\title{
Original
}

\section{An Immunohistological Study on Intraepithelial Lesions in Esophagus}

\section{— Expression of GLUT-1, Cytokeratin, and p53, with Ki-67 Staining -}

\author{
Takahiro Yamazaki, Koji Saito, Tomoaki Mori, \\ Hiromi Date, Shuei Arima, Tomohiko Yoshida, \\ Yuko Date, Mutsuko Omatsu, Tomoko Sugryama, \\ Kaoru Suzuki, Toshiaki Kunimura and Toshio Morohoshi
}

\begin{abstract}
Esophageal epithelial lesions are important features with respect to the early diagnosis of esophageal cancer. Such lesions include inflammatory reactive lesions (inflammatory reactions, IR) and neoplastic lesions. However, while neoplastic lesions are also classified from low-grade intraepithelial neoplasia (LN) to high-grade intraepithelial neoplasia $(\mathrm{HN})$, it is often difficult to differentiate esophageal epithelial lesions in a biopsy. Therefore, this study analyzed cases of endoscopic mucosal resection (EMR) and dissection by immunostaining for p53, MIB-1, glucose transporter-1 (GLUT-1), and cytokeratin $(\mathrm{CK})$ in addition to cytohistological grading to establish lesions trends for classification. This study included 50 lesions of EMR performed at our hospital from 2004 to 2007, as well as 31 cases who underwent a pathological autopsy at our hospital during the period between 2006 and 2007. Tissue diagnoses by HE staining classified the specimens into five groups: normal, IR, $\mathrm{LN}, \mathrm{HN}$, and squamous cell cancer (SCC). This study undertook further comparison by immunostaining for p53, Ki-67 staining, GLUT-1 and CKs 17 and 14. All immunostaining revealed a significant difference between IR and LN grade of lesions. Specifically, GLUT-1 staining revealed a significant difference between $\mathrm{LN}$ and $\mathrm{HN}$ lesions, while CK17 staining differentiated significantly between $\mathrm{HN}$ and SCC. The findings indicated that pathological diagnoses of esophageal lesions should include GLUT-1, CK17, and CK14 immunostaining to differentiate LN and HN lesions.
\end{abstract}

Key words: esophageal epithelial lesions, intraepithelial neoplasia, glucose transporter-1, cytokeratin

\section{Introduction}

Esophageal epithelial lesions are important early indicators of esophageal cancer. Esophageal epithelial lesions include inflammatory reactive (IR) lesions and neoplastic lesions. 
Dysplasia was used to describe intraepithelial tumors in the past, but the term intraepithelial neoplasia was introduced in the WHO classification of $2000^{1)}$. Neoplastic lesions are also differentiated as low-grade intraepithelial neoplasia (LN) or high-grade intraepithelial neoplasia (HN) including cancer in situ by the Japanese Classification of Esophageal Cancer (10th Edition) in Japan ${ }^{2)}$, and different treatments are recommended for $\mathrm{LN}$ and HN lesions, necessitating strict differentiation. However, it is often difficult to differentiate these lesions in a biopsy. In addition, recent advances in diagnostic techniques using magnifying endoscopes and endoscopes ${ }^{3-5)}$ has also defined ultra-microcarcinomas of $1 \mathrm{~mm}^{6}$. These factors and that esophageal cancer is a possible causal factor in elderly cases of dysphagia make early detection even more important.

This study examined the use of immunostaining for p53, Ki-67, glucose transporter-1 (GLUT-1), and cytokeratin (CK) in addition to cytohistological grading to compare and distinguish esophageal lesions.

\section{Materials and Methods}

\section{Subjects}

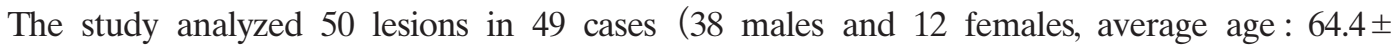
8.9 years) diagnosed as atypical epithelium or squamous cell cancer (SCC) of the esophageal mucosa and who underwent endoscopic mucosal resection (hereinafter referred to as "EMR") at our hospital from 2004 to 2007, as well as 31 control cases (20 males and 11 females, average age : $69.7 \pm 12.6$ years) with no esophageal lesions who underwent a pathological autopsy at our hospital from 2006 to 2007.

\section{Histological study}

Specimens were fixed in formalin and paraffin-embedded, before preparation of sections for hematoxylin and eosin (HE) staining and microscopy. With reference to the Japanese Classification of Esophageal Cancer (10th Edition) ${ }^{2)}$ and various research papers published in Japan ${ }^{7-9)}$, the specimens were diagnosed as normal, IR, LN, HN, or SCC, based on agreement between two pathologists regarding cellular atypia and structural atypia (Fig. 1).

\section{Immunohistochemistry}

Immunostaining was performed to localize p53, Ki-67, GLUT-1, CK17, and CK14 expression. Briefly, sections were deparaffinized and washed, before heat-treatment activation with EDTA ( $\mathrm{pH} \mathrm{8.5)} \mathrm{and} \mathrm{incubation} \mathrm{with} \mathrm{primary} \mathrm{antibody} \mathrm{(DAKO,} \mathrm{Denmark} \mathrm{50-fold} \mathrm{dilution,}$ reaction time: 32 minutes). The sections were then incubated in biotin marker Ig (Ventana Germany) as a secondary antibody and DAB was used to visualize the immunoreaction.

The primary antibodies used were as follows: anti-p53 (DO-7 monoclonal antibody) and anti-Ki-67 (MIB-1 monoclonal antibody) from Dako and used at 1:50 dilution; antiGLUT-1 (polyclonal) from Dako and used at 1: 100; anti-CK14 (polyclonal) from Progen 
Table 1. Immunological study

\begin{tabular}{llccc}
\hline & Maker & Attenuation magnification & origin animal & Production country \\
\hline GLUT-1 (polyclonal) & Dako & $\times 100$ & Rabbit & Denmark \\
P53(DO-7) & Dako & $\times 50$ & Mouse & Denmark \\
Ki-67(MIB-1) & Dako & $\times 50$ & Mouse & Denmark \\
CK14(Polyclonal) & PROGEN & $\times 100$ & Pig & Australia \\
CK17(E3) & Dako & $\times 25$ & Mouse & Denmark \\
\hline
\end{tabular}

(Australia) and used at 1:100; and, anti-CK17 (E3 monoclonal) from Dako and used at 1: 25.

Staining was performed using the Ventana HX System Benchmark automatic staining system (Table 1).

\section{Manifestation characteristics}

We classified p53 and Ki-67 staining by comparison between stainability of p53 and Ki-67 based on Watanabe et $a l^{10)}$. Cases in which Ki-67-positive cells were seen in the more upper layers compared to p53-positive cells, as well as cases in which positive cells were only seen in three rows or lower from the basal side in p53 staining, were classified as type 1 , while cases in which p53-positive cells were seen in 4 to 6 layers from the basal side were classified as type 2, with 7 to 9 layers classified as type 3, and 10 layers or more as type 4 (Fig. 2).

In GLUT-1, cases that were negative or weakly positive in a few layers of the basal portion were classified as type 1 , cases that were strongly positive in a few layers of the basal portion as type 2 , cases that were strongly positive in all layers as type 3 , and cases that were strongly positive in the surface part but negative in the basal side as type 4 (Fig. 3).

In CK14 staining, cases that were strongly positive in 1 or 2 layers of the basal side were classified as type 1 , cases that were strongly positive in a few layers of the basal side as type 2, cases that were positive in all layers as type 3 , and cases that were strongly positive in the surface part but negative in the basal side as type 4 (Fig. 4).

In CK17, negative cases were classified as type 1, cases in which stainability was discontinuous and partially observed were classified as type 2 , cases with continuous staining but not in all layers as type 3, and cases with staining in all layers were classified as type 4 (Fig. 5).

\section{Evaluation method}

Each classification was scored (type 1 is 1 point, type 2 is 2 point, type 3 is 3 points, and type 4 is 4 points). Data were compared using an $\mathrm{m} \times \mathrm{n}$ table $\chi^{2}$ test, with $P<0.05$ considered to indicate a significant difference. 


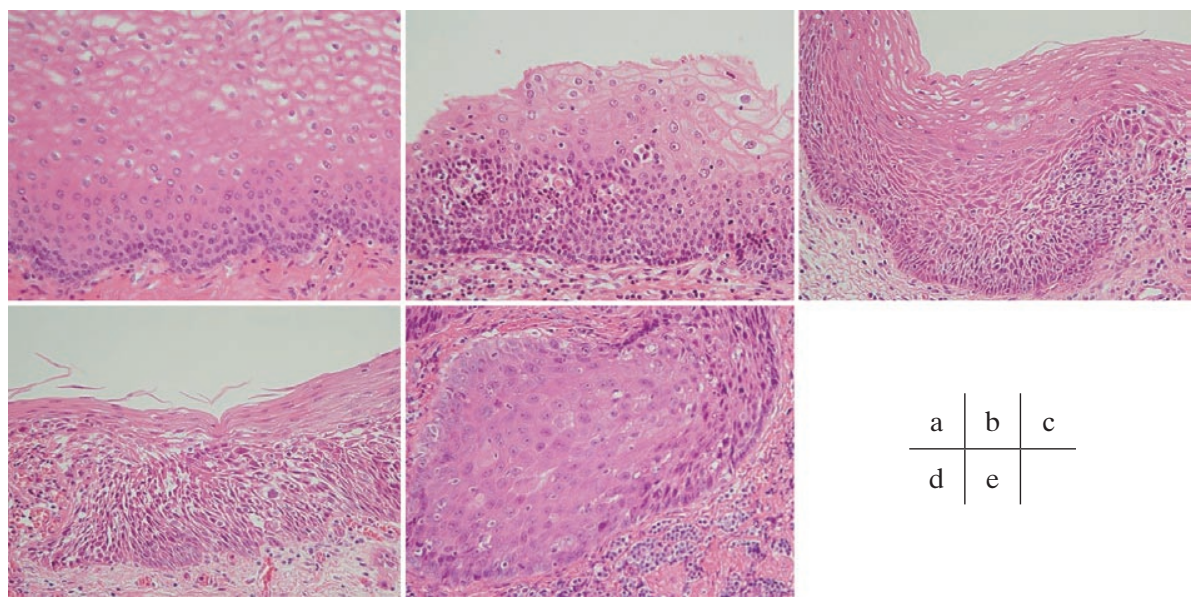

Fig. 1. Examples of lesions classified by HE staining

a. Normal: Only slight inflammatory cell infiltration is observed within the epithelium and the basal cells are finely aligned.

b. IR: The basal cells are slightly out of alignment but no nuclear atypicality or front formation is observed, and inflammatory cell infiltration is observed within the epithelium.

c. LN : Front formation is observed along with nuclear atypicality and increased cell density, but the nuclear atypicality is mild.

d. HN : Compared to LN, the nuclear atypicality is strong and the cell density has increased, but no interstitial infiltration is involved.

e. SCC: Obvious interstitial infiltration is observed.

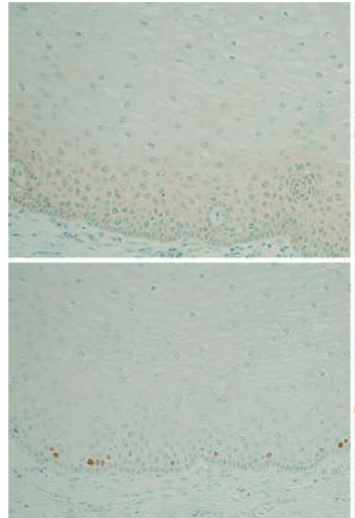

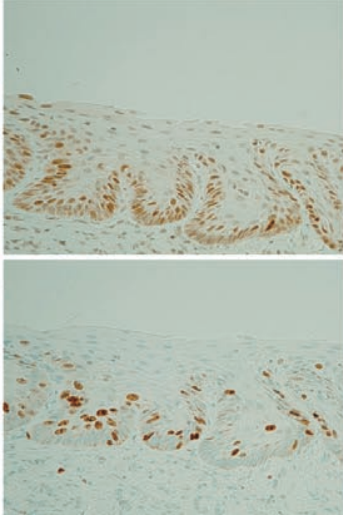

$\mathrm{b}$

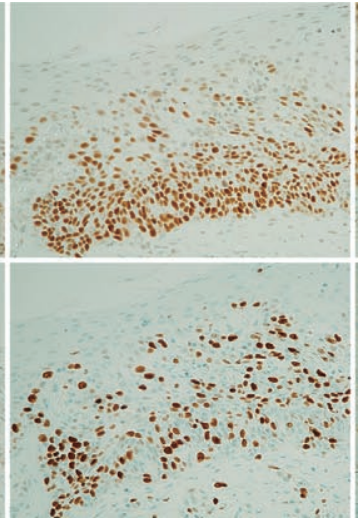

c

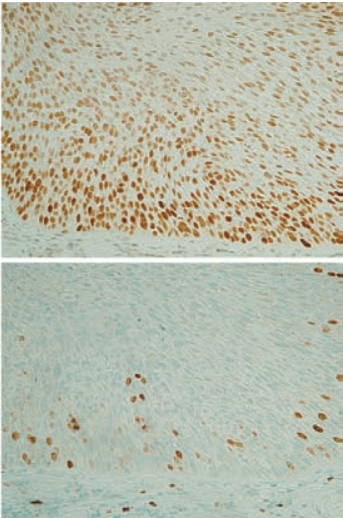

d

Fig. 2. Examples of lesions classified by p53 and Ki-67 staining (upper: p53 ; lower: Ki-67)

a. type 1 ( 1 point) : Ki-67-positive cells are observed in the more upper layers compared to p53-positive cells, or positive cells are observed in only three rows or less from the basal side with p53 staining.

b. type 2 ( 2 points): The stainability of p53 staining is stronger than that of Ki-67 staining, and positive cells are observed in 4 to 6 rows from the basal side with p53 staining.

c. type 3 (3 points) : The stainability of p53 staining is stronger than that of Ki-67 staining, and positive cells are in observed 7 to 9 rows from the basal side with p53 staining.

d. type 4 (4 points): The stainability of p53 staining is stronger than that of Ki-67 staining, and positive cells are observed in 10 rows or more from the basal side with p53 staining. 


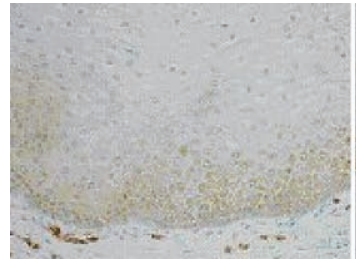

a

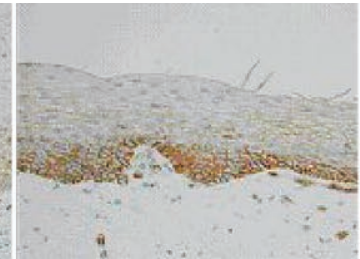

b

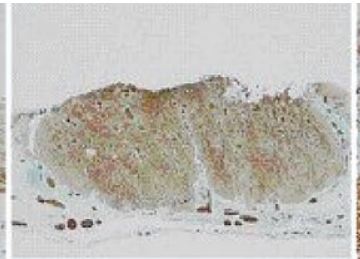

c

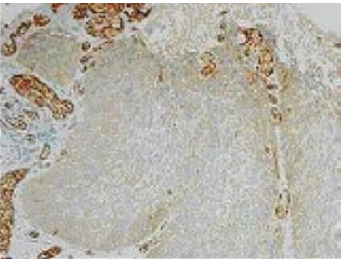

d

Fig. 3. Examples of lesions classified by GLUT-1 staining

a. type 1 (1 point) : Negative or weakly positive in a few layers from the basal side

b. type 2 (2 points) : Strongly positive in a few layers from the basal side

c. type 3 (3 points) : Strongly positive in almost all layers (slightly expanding)

d. type 4 (4 points) : Strongly positive in the surface part but negative on the basal side

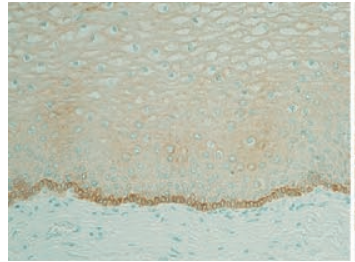

a

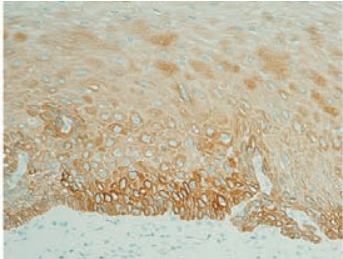

b

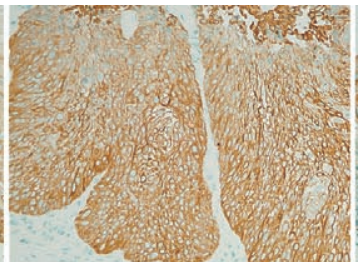

c

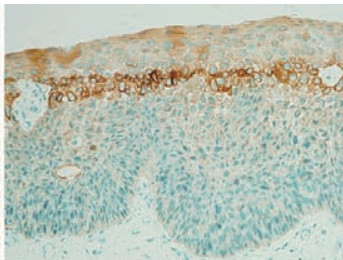

d

Fig. 4. Examples of lesions classified by CK14 staining

a. type 1 ( 1 point) : Strongly positive in 1 to 2 layers from the basal side

b. type 2 ( 2 points) : Strongly positive in a few layers from the basal side

c. type 3 (3 points) : Strongly positive in almost all layers

d. type 4 (4 points) : Strongly positive in the surface part but negative on the basal side

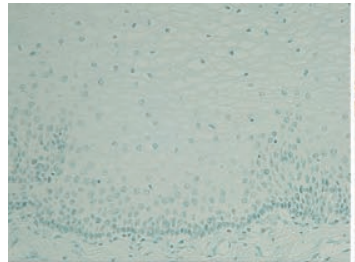

a

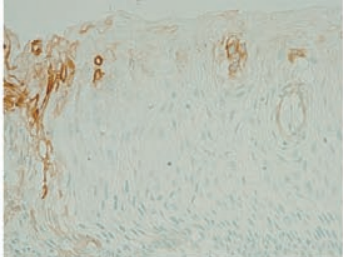

b

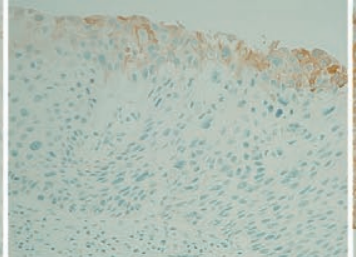

c

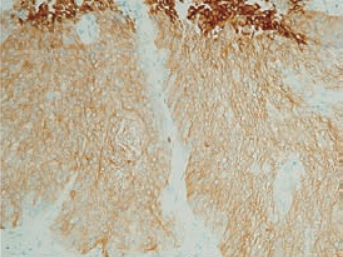

d

Fig. 5. Examples of lesions classified by CK17 staining

a. type 1 (1 point) : Negative

b. type 2 ( 2 points) : A discontinuous positive appearance is observed.

c. type 3 (3 points) : A continuous positive appearance is observed.

d. type 4 (4 points) : Strongly positive in all layers

\section{Results}

Diagnoses of the HE-stained specimens for the total of 81 lesions was normal in 22 subjects, IR in 9 subjects, LN in 11 subjects, HN in 19 subjects, and SCC in 20 subjects.

For p53 and Ki-67 staining, the normal group scored $1.00 \pm 0.00$ point (1 point: 22 subjects), with the IR group at $1.00 \pm 0.00$ point ( 1 point: 9 subjects), the $\mathrm{LN}$ group at 2.73 
Table 2. Frequency of expression of p53/Ki-67

\begin{tabular}{lrrrrc}
\hline & 1 & 2 & 3 & 4 & Ave. \\
\hline Normal & 22 & 0 & 0 & 0 & $1.00 \pm 0.00$ \\
IR & 9 & 0 & 0 & 0 & $1.00 \pm 0.00$ \\
LN & 1 & 3 & 5 & 2 & $2.73 \pm 0.90$ \\
HN & 6 & 0 & 2 & 11 & $2.95 \pm 1.39$ \\
SCC & 5 & 0 & 2 & 13 & $3.15 \pm 1.31$ \\
\hline
\end{tabular}

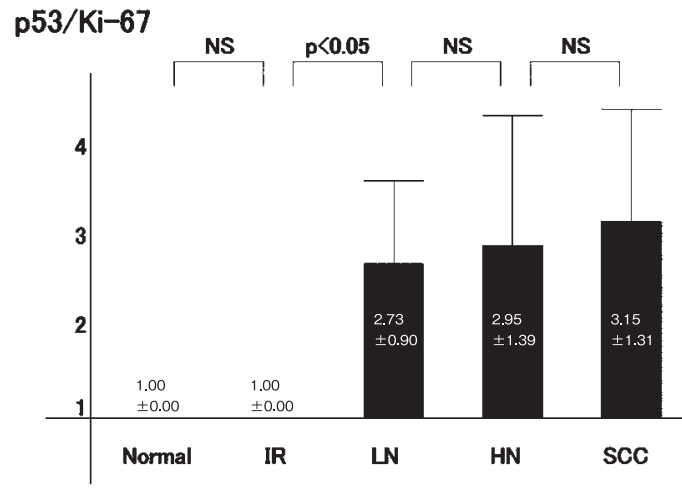

a

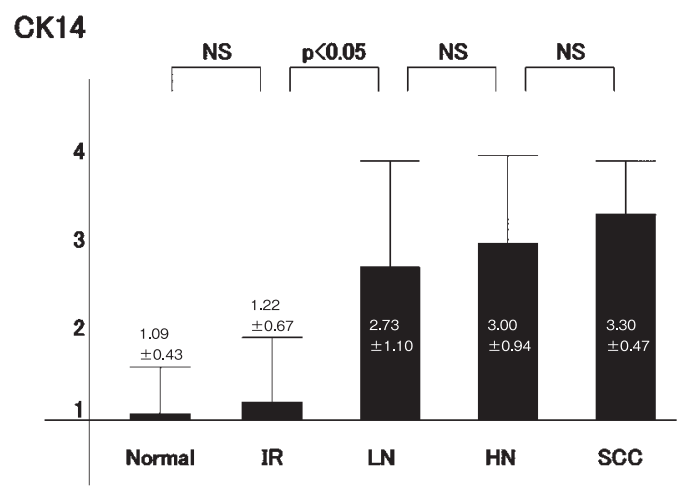

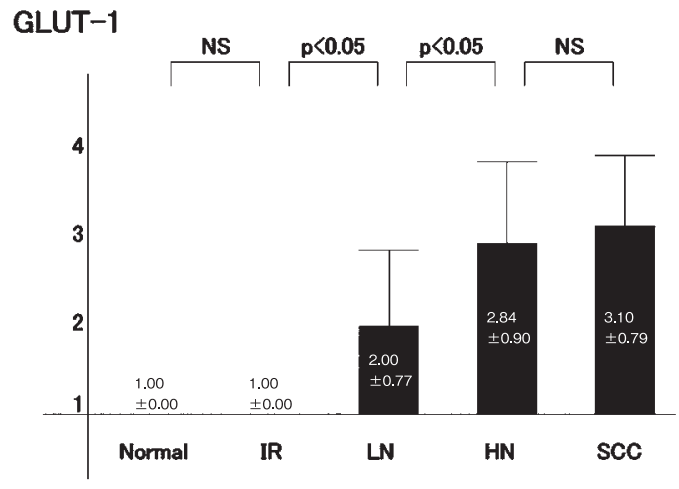

b

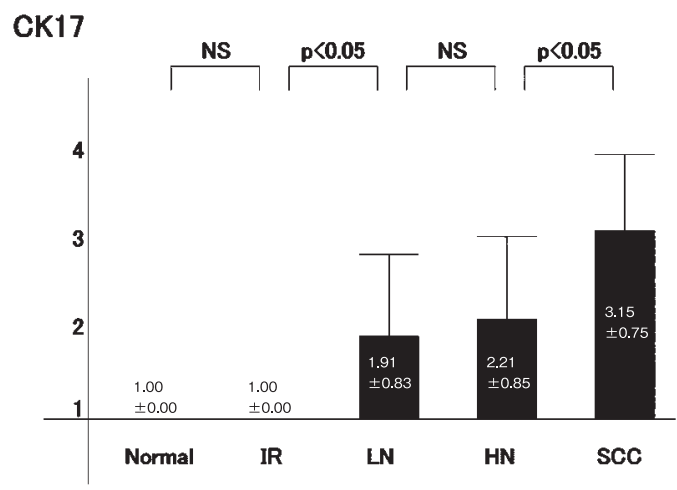

$\mathrm{d}$

Fig. 6. Study of each staining result

\pm 0.90 points (1 point: 1 subject; 2 points : 3 subjects; 3 points : 5 subjects; 4 points : 2 subjects), the $\mathrm{HN}$ group at $2.95 \pm 1.39$ points (1 point: 6 subjects; 3 points : 2 subjects; 4 points: 11 subjects), and the SCC group at $3.15 \pm 1.31$ points (1 point: 5 subjects ; 3 points : 2 subjects; 4 points : 13 subjects) (Table 2). Frequency of expression in the LN group was 2 points or more and therefore significantly higher that those in the IR group (Fig. 6-a) .

For GLUT-1 staining, the normal group scored $1.00 \pm 0.00$ point (1 point: 22 subjects), with the IR group at $1.00 \pm 0.00$ point ( 1 point: 9 subjects), the LN group at $2.00 \pm 0.77$ 
Table 3. Frequency of expression of GLUT-1

\begin{tabular}{lrrrrc}
\hline & 1 & 2 & 3 & 4 & Ave. \\
\hline Normal & 22 & 0 & 0 & 0 & $1.00 \pm 0.00$ \\
IR & 9 & 0 & 0 & 0 & $1.00 \pm 0.00$ \\
LN & 2 & 8 & 0 & 1 & $2.00 \pm 0.77$ \\
HN & 1 & 6 & 7 & 5 & $2.84 \pm 0.90$ \\
SCC & 0 & 5 & 8 & 7 & $3.10 \pm 0.79$ \\
\hline
\end{tabular}

Table 4. Frequency of expression of CK14

\begin{tabular}{lrrrrc}
\hline & 1 & 2 & 3 & 4 & Ave. \\
\hline Normal & 21 & 0 & 1 & 0 & $1.09 \pm 0.43$ \\
IR & 8 & 0 & 1 & 0 & $1.22 \pm 0.67$ \\
LN & 2 & 2 & 4 & 3 & $2.73 \pm 1.10$ \\
HN & 2 & 2 & 9 & 6 & $3.00 \pm 0.94$ \\
SCC & 0 & 0 & 14 & 6 & $3.30 \pm 0.47$ \\
\hline
\end{tabular}

points ( 1 point: 2 subjects; 2 points : 8 subjects; 4 points: 1 subject), the HN group at $2.84 \pm 0.90$ points ( 1 point : 1 subject; 2 points : 6 subjects; 3 points : 7 subjects; 4 points : 5 subjects), and the SCC group at $3.10 \pm 0.79$ points (2 points: 5 subjects; 3 points: 8 subjects; 4 points: 7 subjects) (Table 3). Frequency of expression in the LN group was 2 points or more and therefore significantly higher that in the IR group, but significantly lower than in the HN group, in which the frequency of expression was 3 points or more (Fig. 6-b).

For CK14 staining, the normal group scored $1.09 \pm 0.43$ points (1 point: 21 subjects ; 3 points: 1 subject), with the IR group at $1.22 \pm 0.67$ points ( 1 point: 8 subjects; 3 points : 1 subject), the LN group at $2.73 \pm 1.10$ points (1 point: 2 subjects; 2 points : 2 subjects; 3 points : 4 subjects; 4 points : 3 subjects), the $\mathrm{HN}$ group at $3.00 \pm 0.94$ points ( 1 point : 2 subjects; 2 points: 2 subjects; 3 points: 9 subjects; 4 points: 6 subjects), and the SCC group at $3.30 \pm 0.47$ points ( 3 points : 14 subjects; 4 points : 6 subjects) (Table 4 ). Frequency of expression in the LN group was 2 points or more and therefore significantly higher that in the IR group (Fig. 6-c).

For CK17 staining, the normal group scored $1.00 \pm 0.00$ point (1 point: 22 subjects), with the IR group at $1.00 \pm 0.00$ point (1 point: 9 subjects), the $\mathrm{LN}$ group at $1.91 \pm 0.83$ (1 point: 4 subjects; 2 points : 4 subjects ; 3 points : 3 subjects), the $\mathrm{HN}$ group at $2.21 \pm 0.85$ (1 point: 4 subjects; 2 points : 8 subjects; 3 points: 6 subjects; 4 points: 1 subject), and the SCC group at $3.15 \pm 0.75$ (2 points : 4 subjects; 3 points : 9 subjects; 4 points : 7 subjects) (Table 5). Frequency of expression in the LN group was 2 points or more and therefore significantly higher that in the IR group, while in the SCC group, the frequency of appearance at 3 points or more was significantly higher compared to the HN group (Fig. 6-d) . 
Table 5. Frequency of expression of CK17

\begin{tabular}{lrrrrc}
\hline & 1 & 2 & 3 & 4 & Ave. \\
\hline Normal & 22 & 0 & 0 & 0 & $1.00 \pm 0.00$ \\
IR & 9 & 0 & 0 & 0 & $1.00 \pm 0.00$ \\
LN & 4 & 4 & 3 & 0 & $1.91 \pm 0.83$ \\
HN & 4 & 8 & 6 & 1 & $2.21 \pm 0.85$ \\
SCC & 0 & 4 & 9 & 7 & $3.15 \pm 0.75$ \\
\hline
\end{tabular}

In a comparison of elderly (65 years of age or older) compared to younger patients ( $<65$ years old), 39 subjects (normal : 8 subjects, IR : 3 subjects, LN : 7 subjects, HN : 10 subjects, SCC: 11 subjects) were in the former group, while 42 subjects (normal: 14 subjects, IR: 6 subjects, LN : 4 subjects, HN : 9 subjects, SCC: 9 subjects) were $<65$ years old. Immunostaining showed that the frequency of expression of type 3 and type 4 was significantly high in the elderly cases of the HN group compared to younger cases, but no other significant differences were observed.

\section{Discussion}

According to the WHO classification, lesions localized in half or less of the basal side of the squamous epithelium are classified as LN, whereas those localized in half or more are classified as $\mathrm{HN}^{1)}$. However, in cases where the esophageal epithelium shows epithalaxia or an eroded surface layer, it is difficult to differentiate based on only the height of all of the layers of the esophageal epithelium. Therefore, the Japanese Classification of Esophageal Cancer (Tenth Edition) ${ }^{2}$ ) in Japan recommends that cellular atypia and structural atypia are considered and many research papers in Japan have cited these features for differentiating esophageal epithelial lesions. However, in reality it is not always easy to differentiate these lesions, and there is no consensus among pathologists in Japan ${ }^{7-9)}$. From the perspective of treatment strategy, however, it is very important to differentiate these lesions. For both IR and LN, treatment is not necessary and follow-up is sufficient. However, LN lesions can develop into $\mathrm{HN}$, which might require demucosation, so stricter follow-up is necessary. Recent advances in endoscopic diagnostic techniques such as magnifying endoscopy ${ }^{3-5)}$ have allowed ultra-microcarcinomas of $1 \mathrm{~mm}$ to be detected ${ }^{6)}$, and prognoses have thereby been changed to a large degree by the early diagnoses of esophageal cancer.

Immunostaining for p53 and Ki-67 are commonly used to characterize esophageal epithelial lesions in Japan. Watanabe, et $a l^{10)}$ described that in normal or inflammatory reactive epithelia, p53-positive cells might be found in all three layers from the basal layer, with positive cells in higher layers indicating a high possibility of neoplastic lesion, as does a p53 or Ki-67 marker index of $100 \%$ or higher. However, there are no reports on the value of differentiating among LN, HN, and SCC. In the present study, LN lesions showed a significantly higher rate of p53-positive cells than the IR group, but there were no obvious differ- 
ences in distribution between the $\mathrm{LN}$ group and the $\mathrm{HN}$ group or between the $\mathrm{HN}$ group and the SCC group. This was attributed to the fact that the p53-positive rate in cases of progressive esophageal cancer is approximately $70 \%{ }^{11)}$ and that there were few negative cases in the HN or SCC group in the present study. It was also believed that the distributions became similar because positive cells were observed in ten layers or more in many of the positive cases. Therefore, it is believed that a p53 or Ki-67 marker index is useful to differentiate neoplastic from nonneoplastic cases, but further study is required to differentiate between $\mathrm{LN}$ and $\mathrm{HN}$.

GLUT-1 is part of the glucose transporter family of genetic factors that includes GLUT1 to 5 and 7. GLUT-1 functions as a glucose replacer/ regulator that efficiently conducts glucose transport between the inside and outside of a cell membrane ${ }^{12-14)}$. In the present study, the IR group showed a type 1 staining pattern, but LN lesions were type 2 or higher. It is believed that type 2 or higher staining pattern indicates a high possibility of a lesion of LN or higher. In the HN group, lesions of type 3 and type 4 were observed at high rates, and significant differences were observed in the rates of type $1+$ type 2 and type $3+$ type 4 between the LN group and the HN group $(p=0.004)$. Based on this fact, it is believed that when a strongly positive picture in all layers or negativity on the basal side is observed with GLUT-1 staining, there is a high possibility of a lesion of HN or higher.

GLUT-1 expression is also a marker of tumor hypoxia, tends to be strongly expressed in the center of cancer tissues ${ }^{15,16)}$, is mostly expressed on tumor cells in a hypoxic state, and is observed in a site distant from the blood vessels or in tumor cells around a necrotic focus. Therefore, a pattern in which positivity is exhibited in the tumor cells makes sense, but there are no reports of the basal side becoming negative or any mechanisms proposed. Negativity on the basal side is frequently observed, particularly in high-grade tumors, as is the proliferation of blood vessels around the tumors, indicating the involvement of such negativity. Moreover, GLUT-1 expression in association with cell proliferation, glucose activity, and metabolism has also been indicated, and many cases of positivity in the highgrade atypical epithelium of the uterine cervix, but negativity in the cancer pearl and the significantly cornified cells have been reported ${ }^{17,18)}$. Therefore, it is possible that the basal side exhibits negativity particularly in tumors with a strong tendency toward cornification.

CK is an intermediate filament of 40 to $68 \mathrm{kDa}$ with 20 subunits (CK1 to 20) that differ in molecular weight and isoelectric point by two-dimensional electrophoresis ${ }^{19,20)}$. There are two major types of keratin pairs present in the esophageal squamous epithelium: at $51 \mathrm{kDa}$ and $59 \mathrm{kDa}$, and at $50 \mathrm{kDa}$ and $58 \mathrm{kDa}$, while cancer cells and cultured cells could express other keratin types that do not appear in a normal epithelium, such as $46 \mathrm{kDa}, 48 \mathrm{kDa}$, and $56 \mathrm{kDa}$ forms. These variations indicate that keratin could distinguish esophageal squamous cell cancer from a normal epithelium ${ }^{21,22)}$.

CK14 is equivalent to high-molecular keratin with a molecular weight of $50 \mathrm{kDa}$. It is expressed mainly in basal cells of a normal esophageal stratified squamous epithelium, but 
not in most cases of squamous cell cancer ${ }^{23)}$. In the normal and IR groups in the present study, most of the subjects showed strong CK14 positivity only in 1 to 2 rows of the basal side, in neoplastic lesions with LN or higher, and in lesions of type 2 or higher. It should be compatible that the positive cells which appear in many layers during neoplastic changes may be caused by the proliferation of the basal cells. Moreover, lesions that indicate a pattern such as type 4, in which the basal side exhibits negativity, were occasionally observed, indicating proliferation without the formation of normal keratin. However, no significant differences were observed in distribution between the LN group and the HN group or the HN group and the SCC group, so the value of CK14 for differentiating grades of neoplastic lesions was not apparent.

CK17 is equivalent to low-molecular keratin with a molecular weight of $46 \mathrm{kDa}$ and exhibits negativity both in the basal layer and the surface layer of a normal stratified squamous epithelium ${ }^{24)}$. In the normal and IR groups in this study, all of the subjects were CK17-negative, while positive cells were observed in the neoplastic lesions of LN or higher. Between the LN group and the HN group, similar distributions were observed and no significant differences were observed, but the SCC group lesions exhibited a higher rate of type 4 staining and no negative specimens. As the grade became higher, the stainability increased, indicating that in tumors with a strong tendency toward infiltration, there was a possibility that keratin with a molecular weight that does not appear in a normal esophageal epithelium would appear at a high rate. Indeed, Mituo ${ }^{25)}$ described a keratin with a different molecular weight from the standard form in esophageal squamous cell carcinoma.

In elderly cases, surgery involves high risks and chemoradiation therapy is often selected for progressive cancer. However, if endoscopic treatment is possible as a result of early detection, there is a higher chance of complete cure. Therefore, treatment due to early detection is important, particularly in elderly patients, to affect the prognosis as well as the quality of life. In the present study, few significant differences were observed in stainability between the elderly and nonelderly cases, but such studies should be continued because the number of elderly people undergoing endoscopy will increase due to the aging population, and the number of cases that will be detected at an early stage will increase with more accurate pathological diagnoses.

\section{Conclusion}

Diagnosis of a lesion is important for cases of $\mathrm{LN}$ and $\mathrm{HN}$, because the treatment policies for each condition differ. According to the results of the present study, GLUT-1, CK17, and CK14 immunostaining are useful additions in diagnosing an esophageal lesion. GLUT-1 staining was particularly valuable in differentiating between $\mathrm{LN}$ and $\mathrm{HN}$ lesions.

Performing such immunostaining was thus considered useful for enhancing the diagnostic accuracy of distinguishing lesions for which it is difficult to make a diagnosis due to the small amount of biological material or for lesions in which pathologists cannot reach an 
agreement based on HE staining only.

\section{References}

1) Gabbert HE, Shimoda $T$ and Hainaut $P$ : Squamous cell carcinoma of the esophagus. In: Pathology and Genetics of Tumours of the Digestive System, Hamilton SR and Aaltonen LA (Eds), IARC Press, Lyon, pp 11-19 (2000) (World Health Organization classification of tumours)

2) The Japan Esophageal Society (Ed) : Guide Lines for the Clinical and Pathologic Studies on Carcinoma of the Esophagus, 10th ed, Kanehara \& Co, Tokyo (2007) (in Japanese)

3) Kumagai $Y$, Monma K and Kawada K: Magnifying chromoendoscopy of the esophagus: in-vivo pathological diagnosis using an endocytoscopy system. Endoscopy 36:590-594 (2004)

4) Inoue H, Kazawa T, Sato Y, Satodate H, Sasajima K, Kudo SE and Shiokawa A : In vivo observation of living cancer cells in the esophagus, stomach and colon using a catheter-type contact endoscope, "Endo-Cytoscopy system". Gastrointest Endosc Clin N Am 14 : 589-594 (2004)

5) Inoue H, Kudo SE and Shiokawa A : Technology insight: laser-scanning confocal microscopy and endocytoscopy for cellular observation of the gastrointestinal tract. Nat Clin Pract Gastroenterol Hepathol 2: 31-37 (2005)

6) Inoue H, Sato Y, Kazawa T, Sugaya S, Odaka N, Satodate H, Kudou S and Shiokawa A: Minimally invasive treatment for esophageal diseases - from screening to treatment for 1-mm esophageal cancer involving the ultimate minimally invasive treatment. G.I. Res 12 : 179-189 (2004) (in Japanese)

7) Watanabe G, Ajioka Y, Kobayashi M, Ariga Y, Watanabe Y and Nishikura K: Pathological diagnosis of dysplasia of the esophageal squamous epithelium. Stomach Intest 42 : 137-145 (2007) (in Japanese)

8) Kawachi H: How to read esophageal biopsy specimens - squamous epithelial lesions. G.I. Res 14:79-87 (2006) (in Japanese)

9) Nimura $\mathrm{S}$, Hasuike $\mathrm{N}$ and Shimoda $\mathrm{T}$ : Unstained areas of lugol staining in the esophageal mucosa and the clinicopathological significance thereof from a pathological point of view. Jpn Gastroenterol Surg 30 : 551-560 (2007) (in Japanese)

10) Watanabe H, Nakagawa S, Endo $Y$, Nishikura $K$ and Ajioka $Y$ : Tissue diagnosis of cancer in situ and dysplasia of the esophageal squamous epithelium. Stomach Intest 30:407-416 (1995) (in Japanese)

11) Ohashi K, Nemoto T, Eishi Y, Matsuno A, Nakamura K and Hirokawa K: Proliferative activity and p53 protein accumulation correlate with early invasive trend, and apoptosis correlate with differentiatior grade in oesophageal squamous cell carcinomas. Virchows Arch 430 : 107-115 (1997)

12) Joost HG and Thorens B: The extended GLUT-1 family of suger/polypol transport facilitators: mononclature sequence characteristic and potential function of its novel members. Mol Membr Biol 18 : 247-256 (2001)

13) Medina RA and Owen GI : Glucose transporters : expression, regulation and cancer. Biol Res 35:9-26 (2002)

14) Pessin JE and Bell GI: Mammalian facilitative glucose transporter family: structure and molecular regulation. Аппи Rev Physiol 54 : 911-930 (1992)

15) Doki Y, Takachi K, Ishikawa O, Sasaki Y, Miyashiro Y, Ohigashi H, Yano M, Ishihara R, Tsukamoto Y, Nishiyama $\mathrm{K}$, Ishiguro $\mathrm{S}$ and Imaoka $\mathrm{S}$ : Reduced tumor vessel density and high expression of glucose transporter 1 suggest tumor hypoxia of squamous cell carcinoma of the esophagus surviving after radiotherapy. Surgery 137 : 536-544 (2005)

16) Kato H, Takita J, Miyazaki T, Nakajima M, Fukai Y, Masuda N, Fukuchi M, Manda R, Ojima H, Tsukada K, Kuwano H, Oriuchi $\mathrm{N}$ and Endo K: Correlation of 18-F-fluorodeoxyglucose (FDG) accumulation with glucose transporter (Glut-1) expression in esophageal squamous cell carcinoma. Anticancer Res 23 : 3263-3272 (2003)

17) Matsui N, Yasuda M, Wakui K, Ito J, Hirabayashi K, Kajiwara H, Murakami Y, Sato S and Osamura Y: Significance of glucose transporter-1 (GLUT-1) expression in a region of the uterocervical squamous epithelium. Jpn Soc Clin Cytol 47 : 171-176 (2008) (in Japanese)

18) Mendez L, Manci N, Cantuaria G, Gomez-Marin O, Penalver M, Braunschweiger P and Nadji M: Expression of glucose transporter-1 in cervical cancer and its precursors. Gynecol Oncol 86:138-143 (2002) 
19) Moll R, Franke WW, Schiller DL, Geiger B and Krepler R: The catalog of human cytokeratins: pattern of expression in normal epithelia, tumors and cultured cells. Cell 31:11-24 (1982)

20) Izumi $\mathrm{M}$ : Expression of cytokeratin in various tumors and its application to differential diagnosis. Pathol Clin Med $20: 673-678$ (2002) (in Japanese)

21) Cooper D, Schermer A and Sun TT: Classification of human epithelia and their neoplasms using monoclonal antibodies to keratins : strategies, applications, and limitations. Lab Invest 52 : 243-256 (1985)

22) Grace MP, Kim KH, True LD and Fuchs E: Keratin expression in normal esophageal epithelium and squamous cell carcinoma of the esophagus. Cancer Res $45: 841-846$ (1985)

23) van Dorst EB, van Muijen GN, Litvinov SV and Fleuren GJ : The limited difference between keratin patterns of squamous cell carcinomas and adenocarcinomas is explicable by both cell lineage and state of differentiation of tumour cells. J Clin Pathol 51:679-684 (1998)

24) Troyanovski SM, Guelstein VI, Tchipysheva TA, Krutovskikh VA and Bannikov GA : Patterns of expression of keratin 17 in human epithelia, dependency on cell position. J Cell Sci 93 : 419-426 (1989)

25) Mituo H: Immunohistochemical studies on cytokeratin in the esophageal tissue. Med Bull Fukuoka Univ 18: 147-160 (1991) (in Japanese)

[Received June 30, 2009 : Accepted July 27, 2009] 